УДК 519.1

ОБ ОЦЕНКЕ СТОЙКОСТИ АЕАD-КРИПТОСИСТЕМЫ ТИПА GСМ

\author{
А. Ю. Зубов
}

Московский государственный университет им. М. В. Ломоносова, г. Москва, Россия

Обсуждается методика доказуемой стойкости криптосистем, обеспечивающих конфиденциальность и аутентичность информации. Предлагается упрощённый вариант известной оценки доказуемой стойкости AEAD-криптосистемы GCM с вектором инициализации фиксированной длины. В тех же условиях получена оценка доказуемой стойкости модификации GCM. Приводится сравнительный анализ криптосистем.

Ключевые слова: $A E A D$-криптосистема, GCM, доказуемая стойкость.

DOI $10.17223 / 20710410 / 32 / 4$

\title{
ON THE SECURITY OF AEAD-CRYPTOSYSTEM OF THE GCM TYPE
}

\author{
A. Yu. Zubov \\ Lomonosov Moscow State University, Moscow, Russia \\ E-mail: Zubovanatoly@yandex.ru
}

\begin{abstract}
A provable security methodology for the cryptosystems ensuring information privacy and authenticity is discussed. A simplified version of the well-known estimates for the provable security of the AEAD-cryptosystem GCM with an initialization vector of fixed length is proposed. Under the same conditions an estimate for the provable security of GCM modification is obtained. A comparative analysis of the considered cryptosystems is provided.
\end{abstract}

Keywords: AEAD-cryptosystem, GCM, provable security.

\section{1. Необходимые сведения}

Пусть $G=\{f: S \rightarrow A\}$ - семейство функций, $|S|=k,|A|=n, n<k,|G|=b$. Назовём $G(b ; k, n)$-семейством функиий. Нас будет интересовать свойство функций $f$ из $G$ иметь коллизии, под которыми понимается совпадение $f\left(s_{1}\right)$ и $f\left(s_{2}\right)$ для различных элементов $s_{1}, s_{2}$ из $S$.

Определение 1. Пусть $A=\{0,1\}^{n} ;(b ; k, n)$-семейство $G$ называется $\varepsilon X O R$-yнuверсальным (кратко - $\varepsilon X U$-семейством), если для любых $s_{1}, s_{2} \in S$ и любого $a \in A$ справедливо неравенство

$$
\left|\left\{f \in G: f\left(s_{1}\right) \oplus f\left(s_{2}\right)=a\right\}\right| \leqslant \varepsilon b .
$$

Система MAC типа Wegman - Carter (WC-MAC) основана на $\varepsilon X U$-семействе функций. Известно большое число таких систем. Нам понадобятся введённые в [1] системы $W C[G]$ и $W C[G, F]$. 
Определение 2. Пусть $G=\left\{f: S \rightarrow\{0,1\}^{n}\right\}$-семейство функций и $P=$ $=p_{1}, p_{2}, \ldots$ - случайная равновероятная строка векторов $p_{i} \in\{0,1\}^{n}$. Пусть $\mathrm{cnt}$ - целочисленная переменная (счётчик). Система $W C[G]$ снабжает сообщение $s \in S$ на ключе $\{f, P\}$ меткой $\tau=\left(c n t, p_{c n t} \oplus f(s)\right)$. Для проверки аутентичности сообщения $(s, \tau)$, где $\tau=(i, r)$, вычисляется сумма $p_{i} \oplus f(s)$, которая сравнивается с $r$. Совпадение критерий аутентичности.

Определение 3. Пусть $F:\{0,1\}^{k} \times\{0,1\}^{l} \rightarrow\{0,1\}^{n}$ и $G=\left\{f: S \rightarrow\{0,1\}^{n}\right\}-$ семейства функций. Пусть cnt-целочисленная переменная (счётчик). Система $W C[G, F]$ снабжает сообщение $s \in S$ меткой $\tau(s)=\left(c n t, F_{K}(c n t) \oplus f(s)\right)$, где cnt- текущее значение счётчика, $K \in\{0,1\}^{k}, f \in G$. Ключом служит пара $(K, f)$. При аутентификации каждого следующего сообщения алгоритм генерации МАС увеличивает значение $c n t$ и проверяет условие $c n t<2^{n}-1$. Если неравенство не выполняется, то изменяется значение ключа. Если выполняется, то вычисляется метка по указанной формуле. Для верификации сообщения $(s, \tau(s))$, где $\tau(s)=(i, r)$, проверяющий вычисляет сумму $F_{K}(i) \oplus f(s)$ и сравнивает её с $r$. Равенство - критерий аутентичности.

Реализуемые системами $W C[G]$ и $W C[G, F]$ семейства функций можно рассматривать как псевдослучайные семейства функций (PRF). Мерой их псевдослучайности служит свойство неотличимости случайно выбранного представителя семейства от случайной функции по входам-выходам. Случайной называется функция, значение которой от любого аргумента выбирается случайно и равновероятно из её области значений. Другими словами, это функция, выбранная из семейства функций $\operatorname{Rand}^{S \rightarrow A}$ всех функций $S \rightarrow A$ случайно равновероятно. В рассматриваемом случае $A=\{0,1\}^{n}$. Количественно указанное свойство неотличимости произвольного семейства функций $\Phi=\{f: S \rightarrow A\}$ определяется следующим образом. Проводится атака различения, в которой принимают участие различитель и оракул. Различитель $\mathbf{A}$ (вероятностный алгоритм) может обращаться к оракулу $O^{\varphi}$ функции $\varphi: S \rightarrow A$ с запросами относительно выбранных аргументов $x$ из $S$, получая от оракула значения $\varphi(x)$. На основании полученных ответов А решает, какая гипотеза имеет место:

- $W_{0}$ : функция $\varphi$ выбрана случайно равновероятно из $\operatorname{Rand}^{S \rightarrow A}$;

- $W_{1}$ : функция $\varphi$ выбрана случайно равновероятно из $\Phi$.

Выбор $W_{0}$ или $W_{1}$ произведён заранее, до начала обмена вопросами и ответами.

Результатом работы алгоритма $\mathbf{A}$ является различающий бит $d$, равный 0 , если $\mathbf{A}$ делает выбор в пользу $W_{0}$, и 1 в противном случае. Эффективность работы различителя $\mathbf{A}$ определяется величиной $\mathrm{Adv}_{\Phi, \mathbf{A}}^{\mathrm{prf}}$, называемой $\operatorname{prf-npeuмyществом~семейства~} \Phi$ для различителя $\mathbf{A}$. Она определяется формулой

$$
\operatorname{Adv}_{\Phi, \mathbf{A}}^{\mathrm{prf}}=\left|\mathrm{P}\left[d=1 \mid W_{0}\right]-\mathrm{P}\left[d=1 \mid W_{1}\right]\right|
$$

представляющей собой модуль разности условных вероятностей того, что различающий бит равен 1 , при условии, что имеет место гипотеза $W_{0}$ или $W_{1}$.

При теоретико-информационном подходе затраты различителя не учитываются.

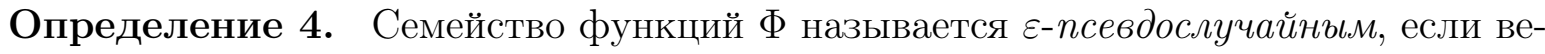
личина

$$
\operatorname{Adv}_{\Phi}^{\mathrm{prf}}=\max _{\mathbf{A}}\left\{\operatorname{Adv}_{\Phi, \mathbf{A}}^{\mathrm{prf}}\right\}
$$

в которой максимум берётся по всем различителям $\mathbf{A}$, удовлетворяет неравенству

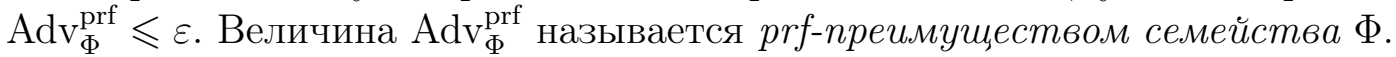


При теоретико-сложностном подходе величина prf-преимущества зависит от объёма затрат. K ним относятся число запросов к оракулу, их общий объём и время работы, под которым понимается действительное время выполнения алгоритма.

Определение 5 . Для любых $t, q, \mu \in \mathbb{N} \operatorname{prf-преимущество~семейства~} \Phi$ - это максимум

$$
\operatorname{Adv}_{\Phi}^{\mathrm{prf}}(t, q, \mu)=\max _{\mathbf{A}}\left\{\operatorname{Adv}_{\Phi, \mathbf{A}}^{\mathrm{prf}}\right\}
$$

по всем различителям $\mathbf{A}$, имеющим временную сложность $t$ и использующим не более $q$ запросов к оракулу, суммарная длина которых не превосходит $\mu$ битов. $\Phi$ называется $\varepsilon(t, q, \mu)$-nсевдослучайным, если выполняется неравенство $\operatorname{Adv}_{\Phi}^{\mathrm{prf}}(t, q, \mu) \leqslant \varepsilon$. Чем меньше $\varepsilon$ (при допустимом объёме затрат), тем «более псевдослучайным» является семейство $\Phi$ (и тем более стойким к атаке различения).

Аналогично определяется prp-преимущество $\mathrm{Adv}_{\Phi}^{\mathrm{prp}}$ семейства подстановок $\Phi$.

Замечание 1. Prf-преимущество семейства функций определяется как prf-преимущество «лучшего» различителя. Если для некоторого различителя выражение под модулем в (1) отрицательно, то для различителя, который принимает противоположный различающий бит, это выражение будет положительным. Второй различитель «лучше» первого. Поэтому, оценивая преимущество семейства функций, можно опускать модуль в определении (1), что обычно и делается.

Замечание 2. Различитель характеризуется набором вероятностей принять различающий бит 1 для каждой последовательности запросов $\bar{x}$ и последовательности $\bar{y}$ ответов на них. Для семейства Rand всех функций для каждого $\bar{x}$ допустим какой угодно $\bar{y}$. «Хороший» различитель семейства $\Phi$ ограничивает для каждого $\bar{x}$ число вариантов $\bar{y}$, для которых указанная вероятность отлична от нуля. Поэтому гипотезам $W_{1}$ и $W_{0}$ отвечают разные распределения вероятностей, $\mathrm{P}_{U}$ и $\mathrm{P}_{V}$, принять различающий бит 1 на множестве $M$ возможных пар $(\bar{x}, \bar{y})$. Пусть для «лучшего» различителя такими распределениями $\mathrm{P}_{U}, \mathrm{P}_{V}$ на (конечном) множестве $M$ определены случайные переменные $U$ и $V$. Тогда, как показано, например, в [2], преимущество «лучшего» различителя можно записать в виде

$$
\max _{f: M \rightarrow\{0,1\}}|\mathrm{P}[f(U)=1]-\mathrm{P}[f(V)=1]|=0,5 \sum_{m \in M}\left|\mathrm{P}_{U}(m)-\mathrm{P}_{V}(m)\right|,
$$

где максимум берётся по всем отображениям из $M$ в $\{0,1\}$. Правая часть этого равенства называется расстоянием по вариации (или статистическим расстоянием) между распределениями $\mathrm{P}_{U}, \mathrm{P}_{V}$. Это равенство даёт другое представление о понятии преимущества.

Определение 6. Система МАС называется $\varepsilon$-стойкой, $0<\varepsilon<1$, если противник, наблюдая ряд аутентифицированных (на одном ключе, выбранном случайно равновероятно) сообщений, может составить новое аутентифицированное (на том же ключе) сообщение с вероятностью, не превосходящей $\varepsilon$.

Утверждение 1. Пусть $G$-это $\varepsilon X U$-семейство функций. Тогда система МАC $W C[G]$ является $\varepsilon$-стойкой.

Доказательство. Пусть противник имеет сообщения $\left(s_{1}, \tau_{1}\right), \ldots,\left(s_{q}, \tau_{q}\right)$, где $\tau_{i}$ метки, полученные системой $W C[G]$ при использовании ключа $(f, P)$. Оценим вероятность того, что противник сможет составить новое сообщение $s^{*}$ и снабдить его меткой $\tau^{*}$, полученной на том же ключе для подходящего значения счётчика $-i^{*}$. 
Противник обязан произвести значение счётчика $-i^{*}$. При этом имеется две возможности: $i^{*} \leqslant q$ и $i^{*}>q$. Если $i^{*}>q$, то $p_{i^{*}}$ - случайный вектор, не коррелирующий с наблюдаемыми значениями. Поэтому сумма $p_{i^{*}} \oplus f\left(s^{*}\right)=\tau^{*}$ также представляет собой вектор, выбранный случайно. Значение $\tau^{*}$ противник может лишь угадать с вероятностью $2^{-n}$. Пусть $i^{*} \leqslant q$. Это означает, что противник выбирает значение счётчика, использованное ранее при вычислении метки $\tau_{i^{*}}$ сообщения $s_{i^{*}}$. Теперь ему нужно произвести $s^{*} \neq s_{i^{*}}$ и $\tau^{*}$, такие, что

$$
\tau^{*}=f\left(s^{*}\right) \oplus p_{i^{*}}=f\left(s_{i^{*}}\right) \oplus p_{i^{*}}=\tau_{i^{*}}
$$

Из этих соотношений получаем равенство $f\left(s^{*}\right) \oplus f\left(s_{i^{*}}\right)=\tau^{*} \oplus \tau_{i^{*}}$.

Пусть $c=\tau^{*} \oplus \tau_{i^{*}}$. Поскольку $G$ образует $\varepsilon X U$-семейство, имеем неравенство

$$
\mathrm{P}\left[f\left(s^{*}\right) \oplus f\left(s_{i^{*}}\right)=c\right] \leqslant \varepsilon .
$$

Таким образом, и искомая вероятность не превосходит $\varepsilon$.

Усилим утверждение 1, предоставив противнику возможность аутентифицировать адаптивно подобранные сообщения и проверить корректность их меток.

Утверждение 2. Пусть $G$ - это $\varepsilon X U$-семейство функций. Тогда при использовании не более чем $q$ запросов к оракулу проверки метки и к оракулу генерации метки система $W C[G]$ является $\varepsilon q$-стойкой.

Доказательство. В серии подделок $\left(s_{i}, \tau_{i}\right), i \geqslant 1$, успех в атаке определяется путём запросов к оракулу проверки метки. Перед каждым таким запросом допускаются запросы к оракулу генерации метки относительно подобранных сообщений.

Пусть $U_{i}$ - событие, означающее неуспех в подделке при $i$-м запросе к оракулу проверки метки. Если имеет место событие $U_{1} \cap \ldots \cap U_{i}$, то, согласно утверждению 1 , вероятность успеха следующей подделки ограничена $\varepsilon$. Поэтому имеет место неравенство

$$
\mathrm{P}\left[U_{i+1} \mid\left(U_{1} \cap \ldots \cap U_{i}\right)\right] \geqslant 1-\varepsilon
$$

Поскольку

$$
\mathrm{P}\left[U_{1} \cap \ldots \cap U_{l}\right]=\mathrm{P}\left[U_{l} \mid\left(U_{1} \cap \ldots \cap U_{l-1}\right)\right] \cdot \mathrm{P}\left[U_{l-1} \mid\left(U_{1} \cap \ldots \cap U_{l-2}\right)\right] \cdot \ldots \cdot \mathrm{P}\left[U_{1}\right]
$$

и каждый сомножитель в правой части не меньше $1-\varepsilon$, получаем неравенство

$$
\mathrm{P}\left[U_{1} \cap \ldots \cap U_{q}\right] \geqslant(1-\varepsilon)^{q} .
$$

Таким образом, вероятность успеха атаки не превосходит величины

$$
1-(1-\varepsilon)^{q} \leqslant \varepsilon q .
$$

Утверждение доказано.

\section{AEAD-криптосистемы, GCM}

В последние годы уделяется большое внимание построению и исследованию новых режимов блочного шифрования, обеспечивающих конфиденциальность и аутентификацию данных [3-7]. В связи с этим возникли новые термины, соответствующие названиям новых режимов шифрования: Authenticated Encryption (кратко - AE) и Authenticated Encryption with Associated Data (AEAD). B AEAD-системах (в отличие от AЕ-систем) используются так называемые ассоииированные данные (associated 
data). Это - дополнительные сведения, которые при передаче должны быть аутентифицированы, но не зашифрованы. Пример - заголовок сетевого пакета, по которому можно определить характер информации, содержащейся в зашифрованном пакете.

AEAD-криптосистема GCM основана на блочном шифровании в режиме счётчика (CTR) и системе аутентификации типа $W C[G, F]$, разработана в 2004 г. [8]. Система GCM стандартизована NIST в документе SP800-38D, имеет доказуемую стойкость, эффективную программную и аппаратную реализацию, широко используется в популярных криптографических протоколах IPSec, MACSec, P1619.1, TLS.

Алгоритм GCM имеет четыре входа:

- ключ $K$;

- вектор инициализации $i v$ длины до $2^{64}$ битов (рекомендуется $i v$ длины 96 битов);

- открытый текст $P$ длины до $\left(2^{32}-2\right) n$ битов ( $n$ - длина блока шифрования);

- ассоциированные данные $A$ длины до $2^{n / 2}$ битов.

Алгоритм GCM имеет два выхода:

- шифртекст $C$, длина которого совпадает с длиной открытого текста;

- метка аутентификации $T$ длины до $n$ битов; длина метки обозначается $\tau$.

Вектор $i v$ должен меняться с каждым открытым текстом. Не требуется, чтобы $i v$ был случайным или непредсказуемым. Он передаётся вместе с шифртекстом. Открытый текст и ассоциированные данные разбиваются на $n$-битовые блоки:

$$
P=P_{1}\|\ldots\| P_{m-1}\left\|P_{m}^{*}, \quad A=A_{1}\right\| \ldots\left\|A_{r-1}\right\| A_{r}^{*} .
$$

Блоки $P_{m}^{*}$ и $A_{r}^{*}$ могут быть неполными. Шифртекст имеет вид $C=C_{1}\|\ldots\| C_{m-1} \| C_{m}^{*}$, где длина блока $l\left(P_{m}^{*}\right)=l\left(C_{m}^{*}\right)$. Пусть

$$
l(P)=(m-1) n+u, 1 \leqslant u \leqslant n ; \quad l(A)=(r-1) n+v, 1 \leqslant v \leqslant n .
$$

Схема алгоритма GCM изображена на рис. 1.

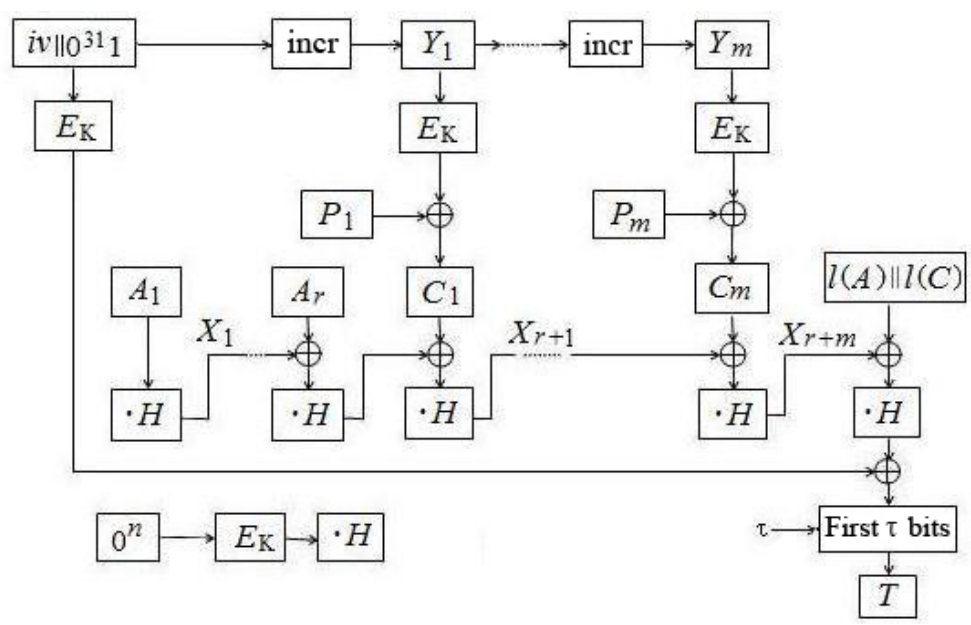

Рис. 1. Схема алгоритма GCM

Алгоритм использует счётчиковую последовательность $Y=Y_{0}\left\|Y_{1}\right\| \ldots$, в которой $Y_{i}=\operatorname{incr}\left(Y_{i-1}\right)$. Функция $\operatorname{incr}(Y)$ изменяет младшие 32 бита $Y$, добавляя единицу по модулю $2^{32}$ :

$$
\operatorname{incr}(L \| R)=L \|(R+1) \bmod 2^{32} .
$$


Алгоритм GCM состоит в выполнении следующих операций:

- $H=E_{K}\left(0^{n}\right)$;

$-Y_{0}= \begin{cases}i v \| 0^{31} 1, & \text { если } l(i v)=n-32, \\ G H A S H(H,\{\}, i v) & \text { в противном случае; }\end{cases}$

- $Y_{i}=\operatorname{incr}\left(Y_{i-1}\right), i=1, \ldots, m$;

$-C_{i}=P_{i} \oplus E_{K}\left(Y_{i}\right), i=1, \ldots m-1$;

$-C_{m}^{*}=P_{m}^{*} \oplus M S B_{u}\left(E_{K}\left(Y_{m}\right)\right)$;

- $T=M S B_{\tau}\left(G H A S H(H, A, C) \oplus E_{K}\left(Y_{0}\right)\right)$.

В описании алгоритма $M S B_{t}(Z)$ обозначает старшие $t$ битов вектора $Z,\{\}$ - пустую строку, $E_{K}(Z)$ - результат зашифрования $Z$ на ключе $K$. Зашифрованное сообщение передаётся в виде вектора $(i v, A, C, T)$. Функция $G H A S H$ определяется формулой

$$
G H A S H(H, A, C)=X_{m+r+1},
$$

в которой $X_{i}, i=0,1, \ldots, m+r+1$, задаются рекуррентным соотношением

$$
X_{i}= \begin{cases}0, & \text { если } i=0, \\ \left(X_{i-1} \oplus A_{i}\right) \cdot H, & \text { если } i=1, \ldots, r-1, \\ \left(X_{r-1} \oplus\left(A_{r}^{*} \| 0^{n-v}\right)\right) \cdot H, & \text { если } i=r, \\ \left(X_{i-1} \oplus C_{i-r}\right) \cdot H, & \text { если } i=r+1, \ldots, m+r-1, \\ \left(X_{m+r-1} \oplus\left(C_{m}^{*} \| 0^{n-u}\right)\right) \cdot H, & \text { если } i=m+r, \\ \left(X_{m+r} \oplus(l(A) \oplus l(C))\right) \cdot H, & \text { если } i=m+r+1,\end{cases}
$$

где операции сложения и умножения выполняются в поле $\mathrm{GF}\left(2^{128}\right)$, порождённом над полем $\mathrm{GF}(2)$ неприводимым многочленом $x^{128}+x^{7}+x^{2}+x+1$.

\section{3. Стойкость GCM}

Оценка стойкости GCM, как и других АЕ-систем, проводится с позиции доказуемой стойкости (provable security). Известна иерархия понятий доказуемой стойкости, отражающих как статистический (или теоретико-информационный), так и теоретикосложностной подходы $[3,6,9,10]$. Второй подход предпочтительнее, поскольку позволяет регулировать выбор параметров криптосистем, обеспечивающих требуемый уровень стойкости. В связи с такой возможностью используется термин практическиориентированная доказуемая стойкость (practice-oriented provable security) [9].

В 2004 г. было показано [10], что для АЕ-систем оценка стойкости типа (IND$\mathrm{CPA})+(\mathrm{AUTH})$ является адекватной мерой стойкости в смысле различных понятий доказуемой стойкости. Понятие (IND-CPA) $+(\mathrm{AUTH})$-стойкости означает следующее.

Стойкость шифрования системы оценивается стойкостью к атаке различения на основе подобранного открытого текста. Стойкость аутентификации системы оценивается стойкостью к активной атаке на основе подобранного открытого текста, в которой атакующий имеет доступ к оракулу генерации метки и оракулу проверки метки. Запросы к оракулам можно как угодно чередовать по усмотрению атакующего. После серии запросов и получения ответов на них атакующий вырабатывает «подделку»пару текст - метка, в которой текст отличен от всех текстов, используемых в запросах. Атака достигает успеха, если метка признаётся корректной. Мерой стойкости служат значения функции преимущества, введённой в определениях 4 и 5 и прокомментированной в замечаниях 1 и 2. В [8] для оценки стойкости GCM использован именно такой 
подход. Приведём доказательство оценки стойкости GCM в случае, когда $l(i v)=n-t$, где $2^{t}$ - максимальное число сообщений, которые можно зашифровать на одном ключе (для GCM $n=128, l(i v)=128-32$ ). В этом случае фактор наличия коллизий функций семейства $G H A S H$ можно не учитывать, что упрощает получение оценки. Этот упрощённый вариант доказательства мы приводим с целью иллюстрации методики.

Теорема 1. Пусть $\mathcal{C}$ - противник, имеющий преимущество $\mathcal{C}_{\mathrm{GCM}}$ в атаке различения семейства функций, реализуемого GCM, или в активной атаке против GCM, при числе запросов к оракулам, не превосходящем $q$. Пусть для каждого запроса $(i v, A, P)$ выполняются условия $l(C)+l(A)<l$ и $l(i v)=n-t$. Тогда существует различитель $\mathcal{B}$ базового шифра $E$, имеющий преимущество $\mathcal{B}_{E}$, где

$$
\mathcal{C}_{\mathrm{GCM}} \leqslant \mathcal{B}_{E}+q\left[\frac{l}{n}+1\right\rceil 2^{-\tau}+\frac{q(q-1)}{2^{n+1}}
$$

Доказательство. Оценим сначала стойкость аутентификации.

Рассмотрим базовый шифр $E$ как псевдослучайное семейство функций (PRF). Пусть $\mathcal{C}$ - противник, имеющий преимущество $\mathcal{C}_{\mathrm{GCM}}$ в активной атаке против $\mathrm{GCM}$. Используем $\mathcal{C}$ для построения различителя $\mathcal{B}$ семейства $E$. В получении ответов на свои запросы $\mathcal{C}$ будет использовать $\mathcal{B}$, направляя ему значения счётчиковой последовательности и получая от него необходимые результаты зашифрования. В свою очередь, $\mathcal{B}$ будет получать эти ответы от оракула зашифрования.

Пусть $W_{1}$ и $W_{0}$ - события, соответствующие использованию в атаке различения для $\mathcal{B}$ семейства функций $E$ или случайной функции. После серии запросов $\mathcal{C}$ строит подделку. Если $\mathcal{C}$ достигает успеха в подделке, то $\mathcal{B}$ делает выбор в пользу $W_{1}$, иначе в пользу $W_{0}$.

Выразим преимущество $\mathcal{B}_{\mathrm{PRF}}=\operatorname{Adv}_{E, \mathcal{B}}^{\text {prf }}$ различителя $\mathcal{B}$ через преимущество $\mathcal{C}_{\mathrm{GCM}}=$ $=\operatorname{Adv}_{\mathrm{GCM}, \mathcal{C}}^{\mathrm{prf}}$ противника $\mathcal{C}$. Согласно $(1)$ и замечанию 1,

$$
\mathcal{B}_{\mathrm{PRF}}=\mathrm{P}\left[d^{\mathcal{B}}=1 \mid W_{1}\right]-\mathrm{P}\left[d^{\mathcal{B}}=1 \mid W_{0}\right],
$$

где $d^{\mathcal{B}}$ - различающий бит, вырабатываемый $\mathcal{B}$. Из описания действий $\mathcal{B}$ и определения преимущества $\mathcal{C}_{\mathrm{GCM}}$ следует, что

$$
\mathcal{C}_{\mathrm{GCM}}=\mathrm{P}\left[d^{\mathcal{B}}=1 \mid W_{1}\right] .
$$

Оценим вероятность $\mathrm{P}\left[d^{\mathcal{B}}=1 \mid W_{0}\right]$.

Лемма 1. $G=G H A S H$ образует $\lceil l / n+1\rceil 2^{-\tau} X U$-семейство функций, где $\tau-$ длина метки и $l$ определяется неравенством $l(A)+l(C) \leqslant l$.

Доказательство. Напомним, что $G H A S H(H, A, C)=X_{m+r+1}$, где $X_{i}$ определяется формулой (2). Рассмотрим два различных входа $(A, C),\left(A^{\prime}, C^{\prime}\right)$ и оценим вероятность события

$$
G(H, A, C) \oplus G\left(H, A^{\prime}, C^{\prime}\right)=a \| z,
$$

где $a$ - фиксированная $\tau$-битовая строка; $z-(n-\tau)$-битовая переменная (отсекаемая часть выхода).

Согласно описанию алгоритма GCM, строки $A, C, A^{\prime}, C^{\prime}$ разбиваются соответственно на $r, m, r^{\prime}, m^{\prime} n$-битовых блоков. Последние блоки имеют длины $v, u, v^{\prime}, u^{\prime}$ соответственно. Пусть $h=\max \left\{m+r, m^{\prime}+r^{\prime}\right\}$ - число блоков в более длинном входе. 
Определим блоки:

$$
D_{i}= \begin{cases}A_{i}, & \text { если } i=1, \ldots, r-1, \\ A_{r}^{*} \| 0^{n-v}, & \text { если } i=r, \\ C_{i-r}, & \text { если } i=r+1, \ldots, r+m-1, \\ C_{m}^{*} \| 0^{n-u}, & \text { если } i=r+m, \\ l(A) \| l(C), & \text { если } i=r+m+1, \\ 0^{n}, & \text { если } i=r+m+2, \ldots, h+1 .\end{cases}
$$

Аналогично определим блоки $D_{i}^{\prime}$ для пары $\left(A^{\prime}, C^{\prime}\right)$.

Равенство (5) можно записать в виде $R(H)=0$, где $R$-многочлен степени, не превосходящей $h+1$, над полем $\mathrm{GF}\left(2^{n}\right)$ :

$$
R(H)=(a \| z) \oplus \sum_{i=1}^{h}\left(D_{i} \oplus D_{i}^{\prime}\right) \cdot H^{i}
$$

Поскольку $A\left\|C \neq A^{\prime}\right\| C^{\prime}$, многочлен $R$ - ненулевой и, следовательно, имеет не более $h+1$ корней. Если $H$ выбирать случайно и равновероятно из $\mathrm{GF}\left(2^{n}\right)$, то вероятность того, что $R(H)=0$, не превосходит $(h+1) 2^{-n} \leqslant\lceil l / n+1\rceil 2^{-n}$ при условии, что суммарная длина входов ограничена $l$ битами.

Нетрудно видеть, что имеется взаимно-однозначное соответствие между блоками $D$ и парами $(A, C)$. Поэтому вероятность того, что $R(H)=0$, для любых двух данных пар $(A, C),\left(A^{\prime}, C^{\prime}\right)$ и данного вектора $a \| z$ равна вероятности события $(5)$. А так как имеется $2^{n-\tau}$ различных значений $z$, событие (5) выполняется с вероятностью, не превосходящей

$$
\lceil l / n+1\rceil 2^{-n} 2^{n-\tau}=\lceil l / n+1\rceil 2^{-\tau}
$$

для любых пар $(A, C),\left(A^{\prime}, C^{\prime}\right)$ и $a \in\{0,1\}^{\tau}$, что и требуется.

Вернёмся к доказательству теоремы. Заметим, что при условии $W_{0}$ система МАС типа $W C[G, E]$ модифицируется в систему МАC типа $W C[G]$, поскольку $E$ заменяется случайной функцией. Такая система МАC, согласно утверждению 1 и лемме 1 , является $\lceil l / n+1\rceil 2^{-\tau}$-стойкой.

Из утверждения 2 получаем неравенство

$$
\mathrm{P}\left[d^{\mathcal{B}}=1 \mid W_{0}\right] \leqslant q\lceil l / n+1\rceil 2^{-\tau} .
$$

Следующее утверждение, известное под названием PRF-PRP-switching lemma [11], связывает $\mathcal{B}_{\text {PRF и }} \mathcal{B}_{\text {PRP }}=\mathcal{B}_{E}$.

Лемма 2. Пусть $\mathcal{A}_{\mathrm{PRF}}\left(\mathcal{A}_{\mathrm{PRP}}\right)$ - преимущество различителя блочного шифра с $n$-битовым блоком и случайной функции (случайной подстановки). Пусть различитель использует не более чем $q$ запросов к оракулу. Тогда

$$
\mathcal{A}_{\mathrm{PRF}} \leqslant \mathcal{A}_{\mathrm{PRP}}+\frac{q(q-1)}{2^{n+1}} .
$$

Утверждение теоремы о стойкости аутентификации следует из (2), (4), (6) и (7):

$$
\begin{gathered}
\mathcal{C}_{\mathrm{GCM}}=\mathcal{B}_{\mathrm{PRP}}+\mathrm{P}\left[d^{\mathcal{B}}=1 \mid W_{0}\right] \leqslant \mathcal{B}_{\mathrm{PRP}}+q\lceil l / n+1\rceil 2^{-\tau} \leqslant \\
\leqslant \mathcal{B}_{E}+q\lceil l / n+1\rceil 2^{-\tau}+\frac{q(q-1)}{2^{n+1}} .
\end{gathered}
$$


Получим теперь оценку стойкости шифрования схемы. Пусть $\mathcal{C}$ - различитель семейства функций, реализуемых $\mathrm{GCM}$, имеющий преимущество $\mathcal{C}_{\mathrm{GCM}}$. Построим различитель $\mathcal{B}$ семейства функций $E$. Для получения ответов на свои запросы $\mathcal{C}$ будет использовать $\mathcal{B}$, направляя ему соответствующие блоки счётчика и получая от него необходимые результаты зашифрования. В свою очередь, $\mathcal{B}$ будет получать эти результаты от оракула зашифрования. Если после серии запросов $\mathcal{C}$ принимает бит 1 , то и $\mathcal{B}$ принимает бит 1.

Пусть $W_{1}$ и $W_{0}$ - события, соответствующие использованию в атаке различения для $\mathcal{B}$ семейства функций $E$ и случайной функции, а $U_{1}$ и $U_{0}$ - события, соответствующие использованию в атаке различения для $\mathcal{C}$ семейства функций GCM и случайной функции. Согласно (1) и замечанию 1 , преимущество $\mathcal{C}$ выражается формулой

$$
\mathcal{C}_{\mathrm{GCM}}=\mathrm{P}\left[d^{\mathcal{C}}=1 \mid U_{1}\right]-\mathrm{P}\left[d^{\mathcal{C}}=1 \mid U_{0}\right] .
$$

В свою очередь, преимущество $\mathcal{B}$ выражается формулой (3).

Из описания действий различителя $\mathcal{C}$ следует, что

$$
\mathrm{P}\left[d^{\mathcal{C}}=1 \mid U_{1}\right]=\mathrm{P}\left[d^{\mathcal{B}}=1 \mid W_{1}\right] .
$$

Оценим вероятность $\mathrm{P}\left[d^{\mathcal{C}}=1 \mid U_{0}\right]$.

Каждый запрос различителя использует счётчиковую последовательность $Y_{0}, Y_{1}, \ldots$ Обозначим через $Q$ событие, означающее, что значения счётчика во всех запросах различны, а через $R$ - событие, означающее, что $\mathcal{C}$ не достигает успеха.

Лемма 3. Справедливо неравенство

$$
\mathrm{P}\left[d^{\mathcal{B}}=1 \mid\left(W_{0} \cap Q\right)\right] \cdot \mathrm{P}\left[Q \mid W_{0}\right] \leqslant \mathrm{P}\left[d^{\mathcal{C}}=1 \mid U_{0}\right] .
$$

Доказателъство. Событие $W_{0} \cap Q$ означает, что в атаке реализуется система $S$, которая получается из GCM заменой функции шифрования случайной функцией, и счётчиковые последовательности в запросах $\mathcal{C}$ не имеют коллизий. Поскольку выход $S$ равносилен выходу случайной функции, событие $\left(d^{\mathcal{C}}=1 \mid\left(U_{0} \cap Q\right)\right)$ означает, что $\mathcal{C}$ принимает случайную функцию за GCM. Отсюда и из того, что $\mathrm{P}\left[U_{0}\right]=\mathrm{P}\left[W_{0}\right]=0,5$, получаем следующие соотношения:

$$
\begin{gathered}
\mathrm{P}\left[d^{\mathcal{B}}=1 \mid\left(W_{0} \cap Q\right)\right] \cdot \mathrm{P}\left[Q \mid W_{0}\right]=\frac{\mathrm{P}\left[\left(d^{\mathcal{B}}=1\right) \cap W_{0} \cap Q\right] \cdot \mathrm{P}\left[W_{0} \cap Q\right]}{\mathrm{P}\left[W_{0} \cap Q\right] \cdot \mathrm{P}\left[W_{0}\right]}= \\
=\frac{\mathrm{P}\left[\left(d^{\mathcal{B}}=1\right) \cap W_{0} \cap Q\right]}{\mathrm{P}\left[W_{0}\right]}=\frac{\mathrm{P}\left[\left(d^{\mathcal{C}}=1\right) \cap U_{0} \cap Q\right]}{\mathrm{P}\left[U_{0}\right]}=\mathrm{P}\left[\left(d^{\mathcal{C}}=1\right) \cap Q \mid U_{0}\right] \leqslant \mathrm{P}\left[d^{\mathcal{C}}=1 \mid U_{0}\right] .
\end{gathered}
$$

Отсюда следует неравенство (10).

Выразим $\mathcal{B}_{\mathrm{PRP}}$ через $\mathcal{C}_{\mathrm{GCM}}$. С учётом $(8)-(10)$ имеем

$$
\begin{gathered}
\mathcal{B}_{\mathrm{PRP}}=\mathrm{P}\left[d^{\mathcal{C}}=1 \mid U_{1}\right]-\mathrm{P}\left[d^{\mathcal{B}}=1 \mid W_{0}\right]= \\
=\mathrm{P}\left[d^{\mathcal{C}}=1 \mid U_{1}\right]-\mathrm{P}\left[\left(d^{\mathcal{B}}=1\right) \mid\left(W_{0} \cap Q\right)\right] \cdot \mathrm{P}\left[Q \mid W_{0}\right]-\mathrm{P}\left[\left(d^{\mathcal{B}}=1\right) \mid\left(W_{0} \cap \bar{Q}\right)\right] \cdot \mathrm{P}\left[\bar{Q} \mid W_{0}\right] \geqslant \\
\geqslant \mathrm{P}\left[d^{\mathcal{C}}=1 \mid U_{1}\right]-\mathrm{P}\left[d^{\mathcal{C}}=1 \mid U_{0}\right]-\mathrm{P}\left[\left(d^{\mathcal{B}}=1\right) \mid\left(W_{0} \cap \bar{Q}\right)\right] \cdot \mathrm{P}\left[\bar{Q} \mid W_{0}\right]= \\
=\mathcal{C}_{\mathrm{GCM}}-\mathrm{P}\left[\left(d^{\mathcal{B}}=1\right) \mid\left(W_{0} \cap \bar{Q}\right)\right] \cdot \mathrm{P}\left[\bar{Q} \mid W_{0}\right] \geqslant \mathcal{C}_{\mathrm{GCM}}-\mathrm{P}\left[\bar{Q} \mid W_{0}\right] \geqslant \\
\geqslant \mathcal{C}_{\mathrm{GCM}}-\mathrm{P}\left[\bar{Q} \mid W_{0}\right]-\mathrm{P}\left[\bar{R} \mid W_{0}\right] .
\end{gathered}
$$


Поскольку условия исключают возможность коллизий в счётчиковых последовательностях, событие $\bar{Q}$ невозможно, и из последних соотношений получаем неравенство

$$
\mathcal{B}_{\mathrm{PRP}} \geqslant \mathcal{C}_{\mathrm{GCM}}-\mathrm{P}\left[\bar{R} \mid W_{0}\right]
$$

Заметим, что в условиях $W_{0}, l(i v)=n-t$ работает система $S$, для которой можно использовать утверждение 2 , согласно которому вероятность $\mathrm{P}\left[\bar{R} \mid W_{0}\right]$ не превосходит $\varepsilon q$, а $\varepsilon$ оценивается в лемме 1 . С учётом этого, из (11) и леммы 2 получаем нужную оценку: $\mathcal{C}_{\mathrm{GCM}} \leqslant \mathcal{B}_{\mathrm{PRP}}+q\lceil l / n+1\rceil 2^{-\tau} \leqslant \mathcal{B}_{E}+q\lceil l / n+1\rceil 2^{-\tau}+\frac{q(q-1)}{2^{n+1}}$.

Теорема доказана.

Приведём числовой пример, иллюстрирующий полученную оценку. Базовым алгоритмом шифрования для GCM является AES. При этом $l(i v)=96, \tau=96, n=128$. Пусть размер сообщения не превышает 1500 байтов (т. е. $l \leqslant 12000$ ). Тогда из теоремы 1 следует, что если AES неотличим от случайной подстановки при защите не более чем $2^{32}$ сообщений на одном ключе (т. е. при этом $\mathcal{B}_{E}$ практически равно 0), то преимущество атакующего AES-GCM не превосходит $5,17 \cdot 10^{-18}$.

\section{4. Оценка стойкости модификации GCM}

Рассмотрим модификацию GCM, в которой система MAC типа $W C[G, F]$ заменяется другой системой WC-MAC. В таких системах вместо $\varepsilon X U$-семейства $G$ может использоваться семейство функций с более слабым требованием к свойству иметь коллизии.

Определение 7. $(b ; k, n)$-семейство функций $G$ называется $\varepsilon$-универсальным $\left(\varepsilon U\right.$-семейством), если для любых $s_{1}, s_{2}$ из $S, s_{1} \neq s_{2}$, и положительного числа $\varepsilon$ справедливо неравенство $\left|\left\{f \in G: f\left(s_{1}\right)=f\left(s_{1}\right)\right\}\right| \leqslant \varepsilon b$.

Определение 8. Пусть $G=\left\{f: S \rightarrow\{0,1\}^{l}\right\}$ и $R=\operatorname{Rand}^{l \rightarrow n}$. Ключом системы МАC $F H[G]$ служит пара $(f, \rho), f \in G, \rho \in R$, а меткой сообщения $s \in S$-значение $\rho(f(s))$.

Определение 9. Пусть $G=\left\{f: S \rightarrow\{0,1\}^{l}\right\}$ и $F:\{0,1\}^{r} \times\{0,1\}^{l} \rightarrow\{0,1\}^{n}$ семейства функций. Ключ системы $F H[G, F]$ - пара $\left(f, F_{K}\right), f \in G, K \in\{0,1\}^{r}$. Меткой сообщения $s \in S$ служит $F_{K}(f(s))$.

У тверждение 3. Пусть $G$ - это $\varepsilon U$-семейство функций и $q$ - максимальное число сообщений, которые можно аутентифицировать на одном ключе. Тогда система МАC $F H[G]$ является $\varepsilon q(q+1) / 2$-стойкой.

Доказательство. Пусть противник наблюдает сообщения $\left(s_{1}, \tau_{1}\right), \ldots,\left(s_{q}, \tau_{q}\right)$, где $\tau_{i}$ - метки, полученные с помощью $F H[G]$ при использовании ключа $(f, \rho)$. Оценим вероятность того, что противник сможет снабдить новое сообщение $s^{*}$ корректной меткой $\tau^{*}$.

Пусть $\Phi=\left\{f\left(s_{1}\right), \ldots, f\left(s_{q}\right)\right\}, T=\left\{\tau_{1}, \ldots, \tau_{q}\right\}$. Если $f\left(s^{*}\right) \notin \Phi$, то $\rho\left(f\left(s^{*}\right)\right)$ с равной вероятностью может принимать любое из $2^{n}$ значений (так как $\rho$-случайная функция). Поэтому вероятность того, что для некоторого $\tau^{*}$ выполняется равенство

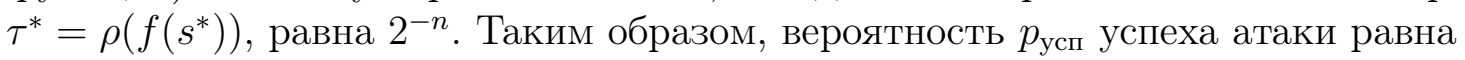

$$
p_{\text {усп }}=2^{-n} \text {. }
$$


Рассмотрим случай, когда $f\left(s^{*}\right) \in \Phi$. В этом случае $\tau^{*}$ должно принадлежать $T$. Тогда в рассматриваемых условиях

$$
p_{\text {усп }}=\mathrm{P}\left[\bigcup_{(i, j)}\left(\tau^{*}=\tau_{j} \mid f\left(s^{*}\right)=f\left(s_{i}\right)\right)\right]=\sum_{i \leqslant j} \mathrm{P}\left[\tau^{*}=\tau_{j} \mid f\left(s^{*}\right)=f\left(s_{i}\right)\right] .
$$

В свою очередь,

$$
\begin{gathered}
\mathrm{P}\left[\tau^{*}=\tau_{j} \mid f\left(s^{*}\right)=f\left(s_{i}\right)\right]=\mathrm{P}\left[\tau^{*}=\tau_{j} \mid f\left(s^{*}\right)=f\left(s_{i}\right), f\left(s_{i}\right)=f\left(s_{j}\right)\right] \cdot \mathrm{P}\left[f\left(s^{*}\right)=f\left(s_{i}\right)=f\left(s_{j}\right)\right]+ \\
+\mathrm{P}\left[\tau^{*}=\tau_{j} \mid f\left(s^{*}\right)=f\left(s_{i}\right), f\left(s_{i}\right) \neq f\left(s_{j}\right)\right] \cdot \mathrm{P}\left[f\left(s^{*}\right)=f\left(s_{i}\right) \neq f\left(s_{j}\right)\right] .
\end{gathered}
$$

Поскольку

$$
\begin{aligned}
& \mathrm{P}\left[\tau^{*}=\tau_{j} \mid f\left(s^{*}\right)=f\left(s_{i}\right), f\left(s_{i}\right)=f\left(s_{j}\right)\right]=1, \\
& \mathrm{P}\left[\tau^{*}=\tau_{j} \mid f\left(s^{*}\right)=f\left(s_{i}\right), f\left(s_{i}\right) \neq f\left(s_{j}\right)\right]=0,
\end{aligned}
$$

получаем формулу

$$
p_{\text {усп }}=\sum_{i \leqslant j} \mathrm{P}\left[f\left(s^{*}\right)=f\left(s_{i}\right)=f\left(s_{j}\right)\right] .
$$

Пусть $D_{i}$ - событие, означающее первое появление коллизии функции $f$ в последовательности аргументов $s_{1}, \ldots, s_{i-1}, s^{*}$ на $i$-м месте. Поскольку $f$ выбирается случайно из $\varepsilon U$-семейства функций, из (13) получаем соотношения

$$
p_{\text {усп }} \leqslant \sum_{i=1}^{q+1} \mathrm{P}\left[D_{i}\right] \leqslant \sum_{i=1}^{q+1}(i-1) \varepsilon=\varepsilon \sum_{i=0}^{q} i=\varepsilon q(q+1) / 2 .
$$

Поскольку $2^{-n} \leqslant \varepsilon \leqslant q(q+1) / 2$, в любом случае из (12) и (13) получаем оценку $p_{\text {усп }} \leqslant \varepsilon q(q+1) / 2$.

Усилим утверждение 3, предоставив противнику возможность аутентифицировать адаптивно подобранные сообщения и проверять корректность их меток. Эти условия могут повысить шансы противника на успех. Покажем, что на самом деле указанные преимущества не позволяют улучшить оценку стойкости, полученную в утверждении 3.

Утверждение 4. Пусть $G$-это $\varepsilon U$-семейство функций. Тогда при использовании не более чем $q$ запросов к оракулу проверки метки и к оракулу генерации метки система $F H[G]$ является $\varepsilon q(q-1) / 2$-стойкой.

Доказательство. В серии подделок $\left(s_{i}, \tau_{i}\right), i=1,2, \ldots, q$, успех в атаке определяется путём запросов к оракулу проверки метки. Пусть $q=q_{1}+q_{2}$, где $q_{1}$ - число запросов к оракулу генерации метки; $q_{2}$ - число запросов к оракулу проверки метки.

Пусть $U_{i}$ - событие, означающее неуспех при $i$-м запросе к оракулу проверки метки, и $p_{i}$ - число запросов к оракулу генерации метки, произведённое перед $i$-м запросом к оракулу проверки метки. Если имеет место событие $U_{1} \cap \ldots \cap U_{i}$, то, согласно утверждению 3 , вероятность успеха в следующей подделке ограничена числом $\varepsilon p_{i+1}\left(p_{i+1}+1\right) / 2$. Это означает, что имеет место неравенство

$$
\mathrm{P}\left[U_{i+1} \mid\left(U_{1} \cap \ldots \cap U_{i}\right)\right] \geqslant 1-\frac{\varepsilon p_{i+1}\left(p_{i+1}+1\right)}{2} .
$$

Поскольку

$$
\mathrm{P}\left[U_{1} \cap \ldots \cap U_{i}\right]=\mathrm{P}\left[U_{i} \mid U_{1} \cap \ldots \cap U_{i-1}\right] \cdot \mathrm{P}\left[U_{i-1} \mid U_{1} \cap \ldots \cap U_{i-2}\right] \cdot \ldots \cdot \mathrm{P}\left[U_{1}\right],
$$


получаем неравенство

$$
\mathrm{P}\left[U_{1} \cap \ldots \cap U_{q_{2}}\right] \geqslant\left(1-\frac{\varepsilon p_{1}\left(p_{1}+1\right)}{2}\right)\left(1-\frac{\varepsilon p_{2}\left(p_{2}+1\right)}{2}\right) \ldots\left(1-\frac{\varepsilon p_{q_{2}}\left(p_{q_{2}}+1\right)}{2}\right) .
$$

Поскольку $p_{i+1} \geqslant p_{i}$ и $p_{q_{2}} \leqslant q_{1}$, вероятность $1-\mathrm{P}\left[U_{1} \cap \ldots \cap U_{q_{2}}\right]$ успеха атаки не превосходит величины

$$
1-\left(1-\frac{\varepsilon q_{1}\left(q_{1}+1\right)}{2}\right)^{q_{2}}
$$

которая, в свою очередь, меньше $\varepsilon q_{1}\left(q_{1}+1\right) q_{2} / 2$.

Так как $q_{1}+q_{2}=q, q_{1} \leqslant q-1$ и функция $q_{1}\left(q_{1}+1\right)\left(1-q_{1}\right)$ принимает максимальное значение при $q_{1}=q-1$, получаем отсюда, что вероятность успеха атаки не превосходит $\varepsilon q(q-1) / 2$, что и требуется доказать.

Рассмотрим AE-систему GCM', которая отличается от GCM лишь тем, что на шаге вычисления метки сумма $G H A S H(H, A, C) \oplus E_{K}\left(Y_{0}\right)$ заменяется результатом зашифрования $E_{K}(G H A S H(H, A, C))$. Это соответствует замене системы $W C[G, F]$ системой $F H[G, F]$.

Как и в теореме 1 , ограничимся случаем, когда вектор $i v$ имеет фиксированную длину. В этих условиях имеет место следующий аналог теоремы 1.

Теорема 2. Пусть $\mathcal{C}$ - противник, имеющий преимущество $\mathcal{C}_{\mathrm{GCM}}$ ' в атаке различения семейства функций, реализуемого GCM', или в активной атаке против GCM', при числе запросов к оракулам, не превосходящем q. Пусть для каждого запроса $(i v, A, P)$ выполняются условия $l(A)+l(C) \leqslant l$ и $l(i v)=n-t$. Тогда существует различитель $\mathcal{B}$ базового шифра $E$, имеющий преимущество $\mathcal{B}_{E}$, где

$$
\mathcal{C}_{\mathrm{GCM}}, \leqslant \mathcal{B}_{E}+\frac{q(q+1)}{2^{n+1}}\left\{\left[\frac{l}{n}+1\right\rceil 2^{n-\tau}+1\right\}
$$

Доказательство. Воспользуемся схемой доказательства теоремы 1. Получим сначала оценку стойкости аутентификации. Пусть $\mathcal{C}$ - противник, имеющий преимущество $\mathcal{C}_{G C M^{\prime}}$ в активной атаке против $\mathrm{GCM}$ ', и $\mathcal{B}$ - различитель семейства функций $E$, построенный так же, как в доказательстве теоремы 1 . Преимущество $\mathcal{B}_{\mathrm{PRF}}$ определяется формулой (3). Из описания действий различителя $\mathcal{B}$ следует, что $\mathcal{C}_{\mathrm{GCM}}$ выражается формулой (4).

При получении оценки вероятности $\mathrm{P}\left[d^{\mathcal{B}}=1 \mid W_{0}\right]$ заметим, что при условии $W_{0}$ реализуется система МАC $F H[G]$. Согласно лемме $1, G=G H A S H$ образует $\varepsilon X U$ семейство функций (следовательно, и $\varepsilon U$-семейство), где $\varepsilon=\lceil l / n+1\rceil 2^{-\tau} ; l(A)+l(C) \leqslant l$.

Из утверждения 4 получаем неравенство

$$
\mathrm{P}\left[d^{\mathcal{B}}=1 \mid W_{0}\right] \leqslant\left\lceil\frac{l}{n}+1\right\rceil \frac{q(q-1)}{2^{\tau+1}} .
$$

Теперь из (8) и (9), с учётом леммы 2, получаем соотношения

$$
\begin{gathered}
\mathcal{C}_{\mathrm{GCM}},=\mathcal{B}_{\mathrm{PRF}}+\mathrm{P}\left[d^{\mathcal{B}}=1 \mid W_{0}\right] \leqslant \mathcal{B}_{\mathrm{PRF}}+\frac{\lceil l / n+1\rceil q(q-1)}{2^{\tau+1}} \leqslant \\
\leqslant \mathcal{B}_{E}+\frac{\lceil l / n+1\rceil q(q-1)}{2^{\tau+1}}+\frac{q(q-1)}{2^{n+1}},
\end{gathered}
$$

откуда следует искомое неравенство. 
Оценка стойкости шифрования GCM' получается так же, как в теореме 1, с той лишь разницей, что в (12) ссылка на утверждение 2 заменяется ссылкой на утверждение 4.

Проиллюстрируем полученную оценку. Пусть длина каждого сообщения не превосходит 12000 битов, $l(i v)=96, \tau=96, n=128$. Тогда преимущество атакующего GCM' не превосходит $1,1 \cdot 10^{-8}$, если базовый блочный шифр неотличим от истинно случайной подстановки при защите не более чем $2^{32}$ сообщений на одном ключе (т. е. если при этом $\mathcal{B}_{E}$ практически равно нулю).

Отметим, что аналогичную оценку стойкости криптосистемы GCM' в общем случае (когда вектор $i v$ имеет произвольную длину) можно получить, используя подход, предложенный в [12].

Как видим, оценку стойкости GCM' можно считать удовлетворительной, хотя она уступает оценке стойкости GCM. Вместе с тем GCM' защищена от атаки Фергюсона [13], которая, по мнению ряда специалистов, представляет собой основную известную угрозу для GCM. Это делает криптосистему GCM' интересной для дальнейшего изучения.

\section{ЛИТЕРАТУРА}

1. Black J. Message authentication codes. PhD Dissertation. Dept. of Comp. Sciences, US Davis, 2000. 126 p. http://www.cs.colorado.edu/ j jrblack/

2. Stinson D. Universal hash families and the leftover hash lemma, and applications to cryptography and computing // J. Combin. Math. Combin. Comput. 2001. V. 42. No. 3. 29 p.

3. Bellare $M$. and Namprempre C. Authenticated encryption: relations among notions and analysis of the composition paradigm // Asiacrypt 2000. LNCS. 2000. V. 1976. P. 541-545.

4. CAESAR: competition for authenticated encryption: security, applicability, and robustness. 2012. http://competitions.cr.yp.to/caesar.html

5. Chakraborty D. and Sarkar P. On modes of operations of a block cipher for authentication and authenticated encryption. Cryptology ePrint Archive: report 627/14. 2014. 51 p.

6. Rogaway P. Authenticated-encryption with associated-data. ACM CCS, ACM Press, 2002. $10 \mathrm{p}$.

7. Svenda P. Basic Comparison of Modes for Authenticated-Encryption (IAPM, XCBC, OCB, CCM, EAX, CWC, GCM, PCFB, CS). 2005. 16 p. https://www.fi.muni.cz/ xsvenda/ docs/AE_comparison_ipics04.pdf

8. McGrew D.A. and Viega J. The security and performance of Galois/Counter mode of operation // LNCS. 2004. V. 3348. P. 343-355.

9. Bellare M. Practice-oriented provable-security // LNCS. 2003. V. 1561. P. 1-15.

10. Shrimpton T. A characterization of authenticated-encryption as a form of chosen-ciphertext security. Cryptology ePrint Archive: 2004/272. 2004. 7 p.

11. Bellare M., Kilian J., and Rogaway P. The security of the cipher block chaining // LNCS. 1994. V. 839. P. 341-358.

12. Iwata T., Ohashi K., and Minematsu K. Breaking and repairing GCM security proofs // Crypto 2012. LNCS. 2012. V.7417. P. 31-49.

13. Ferguson N. Authentication weaknesses in GCM. Public Comments to NIST. http://csrc . nist.gov/CryptoToolKit/modes/comments, May 2005.

\section{REFERENCES}

1. Black J. Message authentication codes. PhD Dissertation. Dept. of Comp. Sciences, US Davis, 2000. 126 p. http://www.cs.colorado.edu/ jrblack/ 
2. Stinson D. Universal hash families and the leftover hash lemma, and applications to cryptography and computing. J. Combin. Math. Combin. Comput., 2001, vol. 42, no. 3. 29 p.

3. Bellare $M$. and Namprempre C. Authenticated encryption: relations among notions and analysis of the composition paradigm. Asiacrypt 2000, LNCS, 2000, vol. 1976, pp. 541-545.

4. CAESAR: competition for authenticated encryption: security, applicability, and robustness. 2012. http://competitions.cr.yp.to/caesar.html

5. Chakraborty D. and Sarkar P. On modes of operations of a block cipher for authentication and authenticated encryption. Cryptology ePrint Archive: report 627/14. 2014. 51 p.

6. Rogaway P. Authenticated-encryption with associated-data. ACM CCS, ACM Press, 2002. $10 \mathrm{p}$.

7. Svenda P. Basic Comparison of Modes for Authenticated-Encryption (IAPM, XCBC, OCB, CCM, EAX, CWC, GCM, PCFB, CS). 2005. 16 p. https://www.fi.muni.cz/ xsvenda/ docs/AE_comparison_ipics04.pdf

8. McGrew D.A. and Viega J. The security and performance of Galois/Counter mode of operation. LNCS, 2004, vol. 3348, pp. 343-355.

9. Bellare M. Practice-oriented provable-security. LNCS, 2003, vol. 1561, pp. 1-15.

10. Shrimpton T. A characterization of authenticated-encryption as a form of chosen-ciphertext security. Cryptology ePrint Archive: 2004/272. 2004. 7 p.

11. Bellare M., Kilian J., and Rogaway P. The security of the cipher block chaining. LNCS, 1994, vol. 839, pp. 341-358.

12. Iwata T., Ohashi K., and Minematsu K. Breaking and repairing GCM security proofs. Crypto 2012, LNCS, 2012, vol. 7417, pp. 31-49.

13. Ferguson N. Authentication weaknesses in GCM. Public Comments to NIST. http://csrc . nist.gov/CryptoToolKit/modes/comments, May 2005. 